\title{
BMJ Open Video game therapy on mobility and dual tasking in multiple sclerosis: study protocol for a randomised controlled trial
}

\author{
Andrea Baroni, ${ }^{1}$ Giulia Fregna, ${ }^{1}$ Giada Milani, ${ }^{2}$ Giacomo Severini, ${ }^{3}$ Giulia Zani, \\ Nino Basaglia, ${ }^{4}$ Sofia Straudi (iD ${ }^{4}$
}

To cite: Baroni A, Fregna G, Milani G, et al. Video game therapy on mobility and dual tasking in multiple sclerosis: study protocol for a randomised controlled trial. BMJ Open 2021;11:e052005. doi:10.1136/ bmjopen-2021-052005

- Prepublication history and additional supplemental material for this paper are available online. To view these files, please visit the journal online (http://dx.doi.org/10.1136/ bmjopen-2021-052005).

Received 02 April 2021 Accepted 23 September 2021

Check for updates

(c) Author(s) (or their employer(s)) 2021. Re-use permitted under CC BY-NC. No commercial re-use. See rights and permissions. Published by BMJ.

${ }^{1}$ Department of Neuroscience and Rehabilitation, Ferrara University Hospital, Ferrara, Italy ${ }^{2}$ Doctoral Program in

Translational Neurosciences and Neurotechnologies, University of Ferrara, Ferrara, Italy

${ }^{3}$ School of Electrical and Electronic Engineering, University College Dublin, Dublin, Ireland

${ }^{4}$ Department of Neuroscience and Rehabilitation, University of Ferrara, Ferrara, Italy

Correspondence to

Dr Sofia Straudi;

s.straudi@ospfe.it

\section{ABSTRACT}

Introduction Multiple sclerosis (MS) is one of the major causes of disability in young adults and affects mobility, compromising daily living activities and participation in social life. Cognitive domain is also frequently impaired in people with MS (PwMS), particularly the capacity to perform dual-task activities. Impaired cognitive processing abilities need to be treated, and motor and cognitive aspects need to be considered together. Recently, video game therapy (VGT) has been used in rehabilitation to improve motor outcomes and cognitive processing speed. The aim of this study is to test the efficacy of commercially available VGT on mobility and dual tasking in PwMS compared with standardised balance platform training (BPT).

Methods and analysis This will be a parallel-assignment, double-blinded, randomised control trial. Forty-eight (24 per arm) PwMS with Expanded Disability Status Scale 4-5.5 will be randomly assigned to receive 1 hour training session over 4 weeks (three sessions/week) of either: (1) VGT on commercial video game console to train balance and mobility-related activities or (2) BPT to perform balance, postural stability and weight-shifting exercises with and without visual feedback. The same assessor will evaluate outcome measures at points: before and after the 12 training sessions and at 3 months of follow-up. The primary outcome will be functional mobility, assessed by the Timed Up and Go test. We will also evaluate gait, risk of fall, fatigue and health-related quality of life as well as cognitive and psychological aspects (depression, anxiety and attentional performance) and stability through posturographic evaluation. Dual-tasking assessment will be performed combining posturographic and neuropsychological tests. Data analysis will be performed to compare the efficacy of the two treatments.

Ethics and dissemination Ethical approval have been granted from the local Ethics Committee. Study results will be communicated through high-quality journals and national and international conferences.

Trial registration number NCT03353974

\section{INTRODUCTION}

Multiple sclerosis (MS) is characterised by multifocal inflammatory demyelinated plaques distributed over time and space within
Strengths and limitations of this study

- This trial will use objective evaluation as a posturographic assessment to test balance and dual tasking.

- Motor and cognitive outcomes will be assessed separately and in association.

- Results from this trial will need a larger sample to be confirmed.

- This study protocol includes only young subjects (aged under 60) with good comprehensive functioning.

- This study does not analyse long-term effects (over 3 months).

the central nervous system (CNS). It affects approximately 1.3 million people worldwide and is a major cause of chronic neurological disability in young adults aged $18-50$ years. ${ }^{12}$ Deficits in balance control and cognition are prevalent impairments in people with MS (PwMS), ${ }^{3}$ even at an early stage and without clinical disability. ${ }^{4}$ Previous studies have reported that $30 \%-63 \%$ of PwMS experience a fall event between 1 and 12 months since the onset of the disease. ${ }^{5}$ Balance maintenance is a complex task that depends on the continuous flow of proprioceptive information from the muscles, tendons, joints, skin, vestibular and visual systems toward the CNS. ${ }^{3}$ In PwMS, the extended damage caused in the CNS leads to a decreased ability in integrating the afferent proprioceptive information, thus negatively influencing postural response and the capability to maintain balance safely. ${ }^{56}$ Balance impairment can consistently limit the activities of daily living and the active participation in social life.

Cognitive impairment is various among PwMS, with current prevalence rates ranging from $43 \%$ to $70 \% .^{7}$ The following cognitive domains are frequently impaired: memory 
processing speed, attention/concentration and executive functioning. ${ }^{8}$ Cognitive impairments are associated with reduced functional status in MS. They have a deleterious impact on the individual's personal, occupational and social functioning and the comprehensive quality of life. ${ }^{9}$

The role of cognitive functioning on motor performance and balance control is widely known. ${ }^{10}$ However, the effects of impaired cognitive processes on balance efficacy have not been extensively investigated in PwMS. ${ }^{11}$ Besides, the comprehensive link between attention and motor-action has been supported by several studies. ${ }^{12}$ Selective attention allows the execution of a correct motor response by selecting relevant information between the task and the distractors, and it is essential for action planning. Concerning the cognitive-motor interference, this relation is directly measurable by the dualtask cost, ${ }^{13} 14$ investigated in PwMS through the Stroop Test. ${ }^{15}$ Dual-task performance is the capacity to do two tasks simultaneously, particularly motor and cognitive tasks. The subject's attention is drawn to an external source of attention while the primary task is ongoing, resulting in cognitive-motor interactions. ${ }^{16}$ Concerning the constrained action hypothesis, this attentional change may lead motor systems to react automatically, thus increasing the performance effectiveness. ${ }^{17}$ The processing capacity required for doing dual-task activities may be affected by cognitive impairments. ${ }^{18}$ In subjects with neurological disorders, such as PwMS, the ability of doing a motor task simultaneously to a cognitive one is frequently affected. ${ }^{17}$

It is plausible that motor deficits enhance the cognitive demands necessary to execute functional movements, and the concurrent performance of tasks may exceed cognitive processing abilities. ${ }^{19}$ Considering the strong impact of dual tasking on activities of daily living (ADL), ${ }^{7}$ rehabilitative treatment does not have to consider the motor and cognitive aspects separately.

In recent years, active video games technologies have begun to be used as a treatment tool in rehabilitation given their low cost, high portability, off-the-shelf nature and ability to deliver engaging, high-repetitive, task-oriented, standardised, active learning therapies. ${ }^{20}$ Moreover, PwMS defined gaming experience as fun, challenging and self-motivating, critical elements for successful motor learning. ${ }^{21}$ Active video games' multisensory feedback provided to patients may potentiate the use-dependent plasticity processes in the sensorimotor cortex, promoting functional recovery. ${ }^{22}$ Furthermore, combined training of cognitive and motor abilities in constantly changing virtual environments is particularly suited to address dualtasking as required for the constantly changing situations of everyday life. ${ }^{23}$

The evidence of interactive video game therapeutic exercises for improving balance and motor functions in PwMS were inconclusive, even if few studies showed a possible positive effect on balance and cognitive functions such as processing speed. ${ }^{24-27}$ Furthermore, patients' motivation seems to be capable of being increased during active video game rehabilitation, allowing patients to exercise more consistently. ${ }^{22}$

All previous studies did not investigate the effects on motor and cognitive functions simultaneously, particularly when compared with conventional instrumental balance training.

\section{Aims}

This study aims to test the efficacy of a commercially available video game therapy (VGT) (Xbox Kinect) on mobility and dual-tasking in ambulatory PwMS in comparison to a standardised balance platform training (BPT).

We hypothesise that augmented feedback during VGT, in terms of intensity and type, would activate the cognitive components of motor learning more effectively than BPT. Moreover, a challenging and engaging approach contributes to enhance treatment adherence and patient's satisfaction.

\section{METHODS AND ANALYSIS \\ Study design and setting}

This is a parallel-assignment, double-blinded, randomised control trial. The outcome assessor and the data analyst will be blinded to the group allocation of participants. PwMS who meet the inclusion criteria and provide written informed consent will be assigned to two treatments, the VGT or the BPT. The trial protocol has been registered on ClinicalTrials.gov.

The protocol of this clinical trial is reported following the Standard Protocol Items: Recommendations for Interventional Trials (SPIRIT) guidelines. $^{28}$ A SPIRIT checklist is available as an additional file (online supplemental additional file 1). Subjects will be recruited from the patients afferent to Outpatient Rehabilitation Clinic at University Hospital of Ferrara. Patients' recruitment started on 1 December 2017, and it is going to finish on 1 October 2022.

\section{Selection criteria and recruitment of participants}

People affected by MS will be included if they meet the following inclusion criteria:

- Men and women, aged 18-60 years;

- Diagnosis of MS (primary or secondary progressive, relapsing-remitting), without relapses in the preceding 3 months;

- Disability rate defined by Expanded Disability Status Scale (EDSS) score from 4 to $5.5^{29}$;

- Balance impairments with increased fall risk, defined as Timed Up and Go (TUG) $>8.4 \mathrm{~s}^{30}$;

- Mini-Mental Status Examination score $\geq 24 / 30 .{ }^{31}$

Exclusion criteria will be:

- Other (neurological) conditions that may affect motor function;

- Visual impairments (daltonism and visual acuity deficit);

- Medical conditions might interfere with the ability to complete the study protocol safely. 


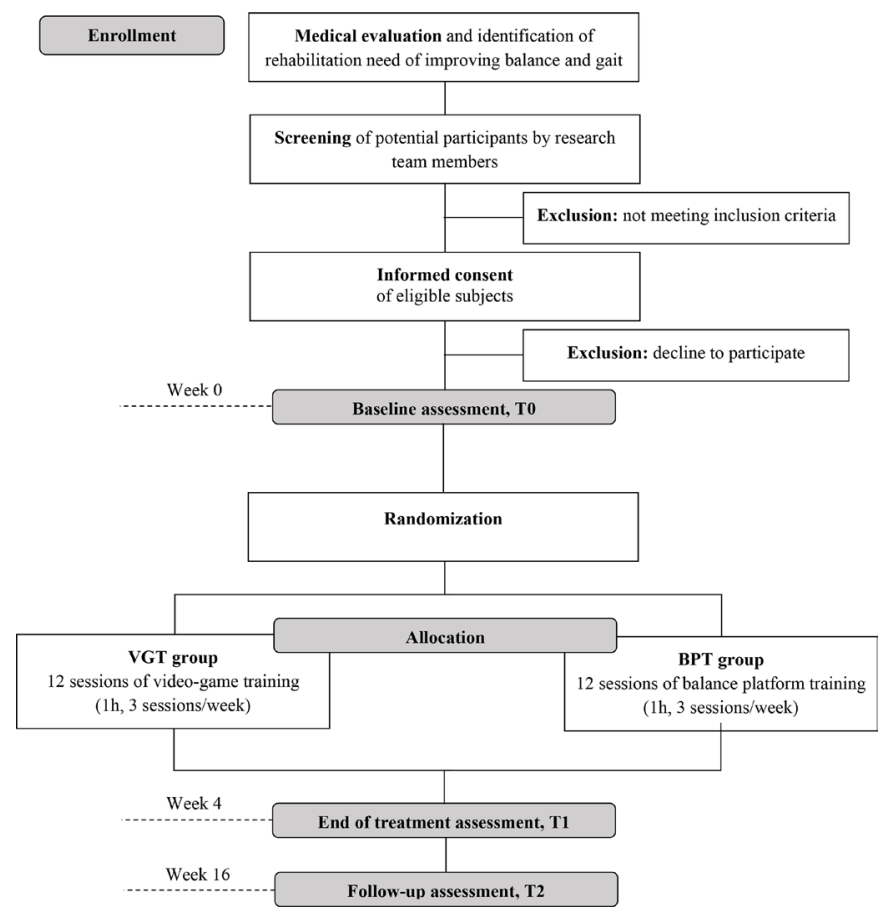

Figure 1 Consolidated Standards of Reporting Trials flow diagram of the study. BPT, balance platform training; VGT, video game therapy.

During the first appointment, potential participants will be informed about all the study procedures and screened following the inclusion criteria, suppose they meet inclusion criteria and are interested in taking part in the study. In that case, the physician will give them a letter explaining study purpose and procedures, time commitments, risks, potential benefits, treatment alternatives, study staff contact information and the Consent Form. A copy of the Consent Form is available as an additional file (online supplemental additional file 2). In the following 3 days, the potential participant will be contacted and asked about its decision; if the subject decides to take part in the study, the research staff will give him/her an appointment for the consign to the signed Consent Form and for the baseline outcome measures, conducted by a physiotherapist. If the subject rejects participation in the study, the research staff remains available for further information. The total number of subjects screened for participation and the number of subjects who decline to participate will be recorded, according to the Consolidated Standards of Reporting Trials (CONSORT) guidelines (figure 1). ${ }^{32}$

\section{Randomisation and blinding}

People meeting inclusion criteria who decided to participate will be assigned to one of the two treatment groups through a block randomisation approach (1:1 ratio). The randomisation scheme will be set up in permuted blocks of four to ensure a similar number of participants between groups. The randomisation scheme will be generated using the website http://www.randomization. com and managed by an external administrator to the research group to prevent selection bias. The outcome assessor will be blinded about the subject's group allocation. All outcome data and assignment groups will be organised in different data sets to maintain the blinding during data analysis. The privacy of the participants and their medical records will be guaranteed by treating the data according to the Italian Law n. 196/2003, to the 'Safe Harbor Act' (2000/520/CE) and to the 'European Union Data Protection Directive (95/46/EC 24 October 1995)'.

\section{Intervention}

All participants will receive twelve 1-hour training sessions over 4 weeks, resulting in a three sessions/week scheme. To manage possible absence lasting one or more treatment sessions, a potential window of 5 weeks will be set to ensure the achievement of all 12 sessions. Subjects who miss a training session will be contacted by phone to determine the absence's reasons and maintain adherence to following treatment sessions. Subjects who miss more than three consecutive treatment sessions will be excluded from the study.

Every training session consists of about $50 \mathrm{~min}$ of exercise and about $10 \mathrm{~min}$ of mobility and flexibility activity to prevent muscle soreness due to movement. All interventions will be delivered at the Rehabilitation Clinic of the University Hospital of Ferrara.

\section{VGT}

VGT will be delivered with a commercial video game console, Kinect for Xbox 360 game system (Microsoft Corporation, Redmond, Washington, USA). Pre-selected games were chosen from 'Kinect Adventures!' and 'Kinect Sports' (Microsoft Game Studios) that encompassed a wide range of motor activities in a standing position. Specifically, balance and mobility-related motor tasks were trained, such as sidestepping, lateral weight shifting, jumping, walking (lateral, forward and backward), and arm goal reaching.

Kinect is a commercial device therefore calibration of the instrument on the individual patient is not provided. The physiotherapy treatment with this device will be set by the physiotherapist basing on clinical observations of the patient's characteristics, preferences and level of functioning.

A list of games will be tested according to the patient's abilities and preferences. In the following sessions, games will be proposed with a randomised practice approach. Progression proceeds over time according to the patients' motor and balance improvements. Each task will consist of 2-5 min of training and a rest period will be given if necessary. During sessions, the patients will be carefully supervised by a physiotherapist who monitored the patient's safety (eg, risk of falls, impulsive reactions). The physiotherapist will also give performance feedback and those provided by VGT: visual and augmented (knowledge of both results and performance). ${ }^{33}$ Despite variability among treatment protocols available in the literature, our treatment dosage in terms of number of 
sessions and intervention duration is in line with other studies on the efficacy of exergame in people with neurological disorders. ${ }^{2534-36}$

\section{BPT}

Balance/rebalancing, postural stability and weightshifting exercises with and without visual feedback will be administered using a Balance Platform (Biodex Medical Systems, Shirley, New York, USA) that had been previously tested in patients with MS. ${ }^{37}$ Each task will be trained for about 2-3 min, followed by a rest period when necessary. During the first session, the tasks will be set to an 'entry-level'. The exercise progression will be adjusted over time according to the patient's capabilities (intermediate and difficult level). BPT offers visual feedback about reaching goals (augmented feedback). The physiotherapist will carefully supervise the patient and monitor his safety, providing additional external feedback during the activities.

\section{Concomitant care and recommendations}

All the subjects will be advised to not undertake other physical treatments until the end of the assessment period. Subjects will also be encouraged to not use the video game console at home for leisure to prevent confounding effects. It will be asked to patients to wear the same shoes and orthosis during all the outcome assessment and training sessions.

\section{Intervention fidelity and monitoring of adverse events}

All the interventions will be delivered by a physiotherapist with at least 5 years of experience in the treatment of PwMS, properly formed about VGT or BPT. Training sessions features and comments will be tracked in a precompiled form. Any unpredictable adverse events will be recorded in the patient's registry and the electronic study database. Their management will agree with the related hospital policies, with a referral for appropriate medical follow-up.

\section{Outcome assessment and data collection}

All the clinical evaluations will be performed at the Ferrara University Hospital by the same blinded assessor at the three time-point evaluations: (T0) baseline, before the first intervention; (T1) end of treatment, after the 12 therapy sessions; (T2) follow-up, 3 months after the end of treatment. Clinical and posturographic assessment will be delivered by a physiotherapist trained adequately about evaluation procedures. A neuropsychologist with years of experience in the assessment and treatment of PwMS will provide cognitive tests. A physician member of the research team will define the patient's EDSS score. A team member will record the general demographic information (age, gender), comorbidities and medical history of every participant. A summary of the study plan is reported in table 1.

\section{Primary outcome: functional mobility}

This function will be assessed by the TUG test, a reliable and valid performance-based measure of functional mobility. ${ }^{30}$ The patient will be instructed to stand up from a chair, walk for $3 \mathrm{~m}$, cross a line marked on the floor, turn around, walk back and sit down. The time used to complete the task is recorded using a chronometer. During the assessment, the subject can use any necessary gait aid (not physical assistance). This test will be the first performed during the assessment session to reduce variability due to the subject's fatigue. The TUG test

Table 1 Schedule of enrolment, interventions and assessments

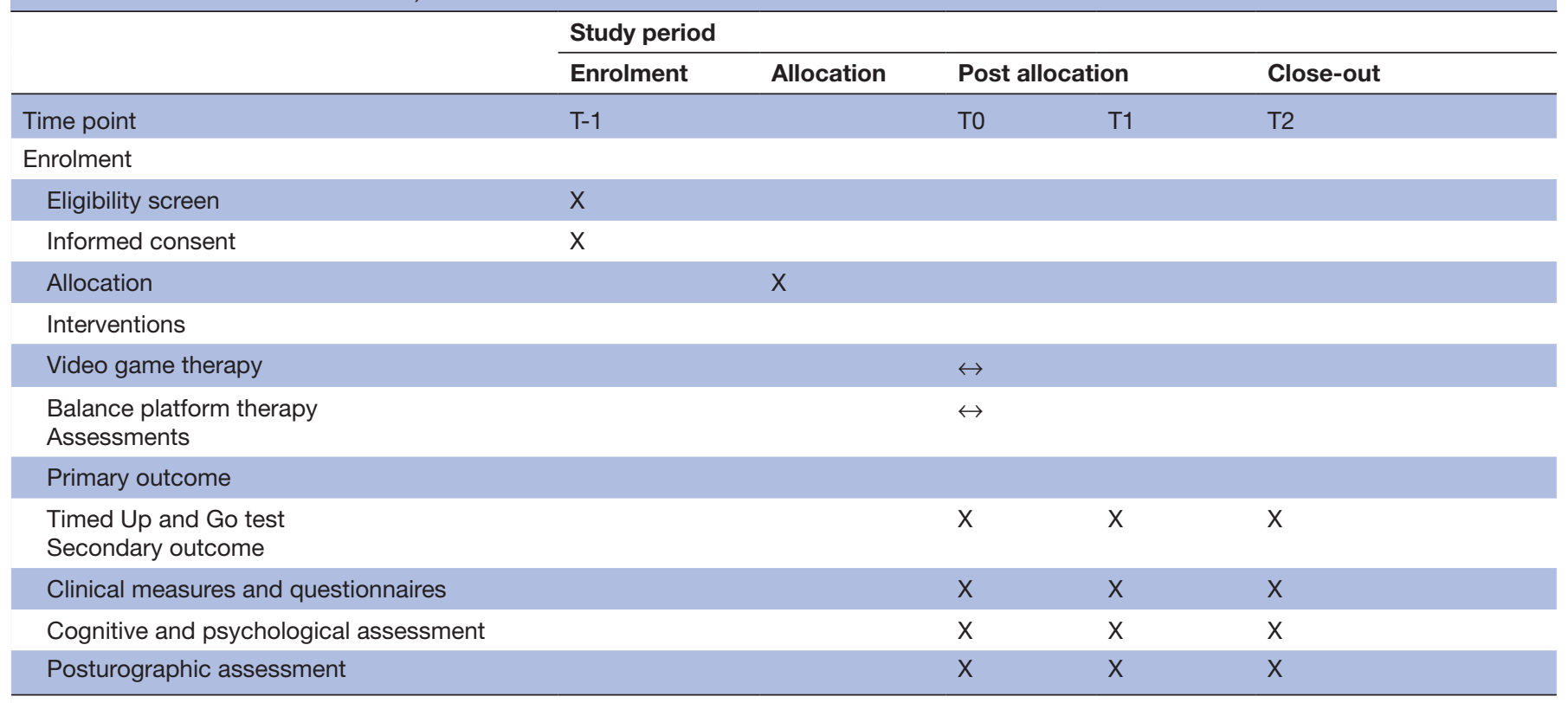

T-1 enrolment, T0 before treatment, T1 after treatment, T2 3-month follow-up. 
will be repeated three times, and the mean value will be recorded.

\section{Secondary outcome measures}

Secondary outcomes will include: (1) clinical measures and questionnaires, (2) cognitive and psychological assessment and (3) posturographic assessment. All secondary outcome measures will be carried out in random order.

\section{Clinical measures and questionnaires}

- Dynamic Gait Index (DGI): gait, balance and risk of fall are measured using DGI. DGI will evaluate not only usual steady-state walking but also during a more challenging task (ie, cross obstacles, slalom). The subject will perform eight functional walking tests and score out of three (maximum total score is 24$).{ }^{38}$

- Four Square Step Test (FSST): this timed test is intended to challenge the rapid change in direction while stepping forward, backward and sideways over a low obstacle. The faster the time measured to perform the task signifies a superior level of dynamic balance abilities. The minimal detectable change estimate for the FSST in MS is $4.6 \mathrm{~s}$, and it was found to be a valid assessment tool in MS. ${ }^{39}$

- Functional Reach Test (FRT): this test assesses the subject's stability by measuring the maximum distance an individual can reach forward while standing in a fixed position. A longer reaching distance indicates better postural control. ${ }^{40}$

- Multiple Sclerosis Impact Scale-29 (MSIS-29): this health-rated quality of life questionnaire assesses the impact of MS on physical and psychological functions. It is formed by 29 items on ADL I and II: 20 about physical activity and 9 about psychological status. Each item can be scored with a value from 0 to 5 ; the total score is given by the sum of all the items and then is transformed in a range from 0 to $100 .^{41}$

- Multiple Sclerosis Walking Scale-12 (MSWS-12): this questionnaire assesses the impact of MS on walking ability. It is formed by 12 items, asking the patient about his perception on gait speed, running, confidence ascending/descending stairs, balance and fatigue. The total score is obtained by the sum of the score of each item (0-5) and then transformed into a value from 0 to $100 .{ }^{42}$

- Modified Fatigue Impact Scale (MFIS): this 21-item questionnaire assesses the perceived impact of fatigue on the subscale physical, cognitive and psychological functioning during the past 4 weeks. MFIS has been recommended for use by the Multiple Sclerosis Council for Clinical Practice Guidelines. ${ }^{434}$

\section{Cognitive and psychological assessment}

- Beck Depression Inventory-Second Edition (BDIII): this is a 21-item self-report measure that quantifies the severity of depression and over behavioural characteristics of depression. ${ }^{45}$
- State Trait Anxiety Inventory (STAI-Y): this self-report questionnaire measures the presence and severity of current symptoms of anxiety and a generalised propensity to be anxious. There are two subscales: 20 items allocated to each of the State Anxiety (SAnxiety) and Trait Anxiety (T-Anxiety). ${ }^{46}$

- Test of Attentional Performance (T.A.P. V.2.3): attentional performance will be evaluated using a neuropsychological computer test. Errors, omissions and reaction times will be recorded as outcomes of performance. Three modules of the T.A.P. will be administered: Go-No Go subtest as it allows assessment of the specific ability of subjects to suppress undesired responses; alertness subtest that measures the simple reaction time in response to a visual stimulus and, divided attention subtest, that explored with 'dual-task' test the ability to attend simultaneously two stimuli (visual and acoustic) processed in parallel. ${ }^{47}$

- Stroop Color-Word Test (SCWT): this neuropsychological test is used to assess the ability to inhibit cognitive interference when processing a specific stimulus feature impedes the simultaneous processing of a second stimulus attribute. Subjects are required to read three different tables as fast as possible. Two of them represent the 'congruous condition', in which participants are required to read names of colours printed in black ink and name different colour patches. Conversely, the third table represents the incongruent condition, in which participants are required to name the colour of the ink instead of reading the word. ${ }^{48}$

- Symbol Digit Modalities Test (SDMT): this neuropsychological test quantifies cognitive processing speed. It consists of orally report the correct number corresponding to a symbol in a pseudorandom sequence of nine symbols as quickly as possible. ${ }^{49}$

\section{Posturographic assessment}

Instrumented basic balance evaluation: Force platform measurements are routinely used as objective markers of subjects' balance ability. ${ }^{50}{ }^{51}$ Several parameters can be extracted from the force platforms that correlate with balance ability and risk of falls in PwMS. During the instrumented tasks, subjects will be asked to stand on a force plate (BERTEC Model 4080-10, Bertec, Columbus, Ohio, USA) with arms parallel to their body. Each subject will undergo five repetitions (each lasting $60 \mathrm{~s}$ ) of two tasks that will consist in standing with the eyes open and standing with the eyes closed. The movements of the centre of pressure $(\mathrm{CoP})$ of the subjects will be recorded. A series of features will be extracted from the CoP traces to inform on different balance characteristics of each patient and the specific effect of each of the proposed therapies. Features that will be analysed include different measures of CoP displacement in the anteroposterior (AP) and mediolateral (ML) directions, the CoP path length (total, AP and ML) and the CoP average and maximum speed. ${ }^{33}$ 
Dual-tasking assessment will be performed combining posturographic and neuropsychological tests: subjects will be asked to complete SCWT standing on the force platform, ensuring a similar condition to single-task SCWT. Head position will be fixed using a large panel fixed on the wall facing the force plate in order to prevent subjects lowering their head and distort the data. The ability to inhibit cognitive interferences during quiet and standing conditions will be compared.

\section{Data management}

Data analysis will be performed according to the research hypothesis mentioned. Stata Statistical Software (Release 13.1.: StataCorp LP) will be used for data analysis.

\section{Sample size and power}

The primary outcome of this study is to highlight differences in the time used to perform the TUG test between PwMS who underwent VGT and BPT. Our preliminary results from an unpublished pilot study $(n=6)$ shown a VGT effect size of 0.93 in people with MS and EDSS $<6$. Given equal allocation between treatment and control arms, and using $80 \%$ power and alpha of $5 \%$, we would need 40 subjects to complete the study. Conservatively, we expect a $10 \%$ rate of drop-out. Thus the sample size will be increased by $10 \%$ to 48 subjects ( 24 per arm).

\section{Statistical analyses}

Descriptive statistics (mean and 95\% CI) will be reported before treatment, after treatment and at 3 months follow-up for all the selected variables (clinical, instrumental and questionnaires). Specifically, TUG changes after treatments will be considered as our primary endpoint, whereas changes of all the other outcome measures (DGI, FSST, FRT, MSIS-29, MSWS-12, MFIS, BDI-II, STAI-Y, SCWT, SDMT, T.A.P., posturographic variables) will be treated as secondary endpoints. Betweengroup differences will be explored with the Wilcoxon rank-sum test. Moreover, a repeated-measures analysis of variance (within-group factor: TIME; between-group factor: TREATMENT) will be conducted to detect the main effects for treatment and time for all the available outcomes. To calculate the effect size of both treatments, we will use Cohen's $d{ }^{52}$ Results will be reported as mean and $95 \%$ CI. Significance will be recognised for $\mathrm{p}<0.05$.

\section{Intention-to-treat}

Every attempt will be made to avoid missing data through a careful check of self-reported measures, as self-administered questionnaires. An intention-to-treat analysis was carried out on all outcome measures. Missing data will be treated using the last observation carried forward approach.

\section{Data monitoring and interim analysis}

The trial does not include Data Monitoring Committee. An update on trial progress will be shared with Ferrara University Hospital Research office every 6 months. The research coordinator will be responsible for interim analysis to determine if the trial should stop, modify or carry out. The research group will discuss any subsequent modifications and communicate to the funding agency and Ethics Committee.

\section{Patient and public involvement}

The research question in this study starts from years of experience in rehabilitation of PwMS and previous research study on the use of VGT in balance impairment. The drafting of the study was submitted to the Multiple Sclerosis Italian Society (FISM) and reviewed to better meet patients' needs. The final version of the study reflects the collaboration between the research group and the patients' association.

\section{Ethics and dissemination}

The Ethics Committee of Ferrara approved this study with approval number 170691 on 19 October 2017. Communication of results and conclusions will be assigned to high-quality journals and national and international conferences. Results will also be disseminated through FISM annual conference. People with MS will be informed about the possible efficacy of the proposed treatment through MS support groups.

\section{DISCUSSION}

This trial may highlight the role of gaming in the rehabilitation of PwMS, enforcing the utilisation of new technologies in daily clinical practice among subjects with mild-to-moderate disability. ${ }^{53}$

Our expectation from the proposed research is to observe a more significant effect on mobility, balance and dual-tasking through virtual reality training compared with a conventional approach. A meta-analysis by CasusoHolgado et al found that active video game training could be considered as effective as conventional training in improving balance and gait abilities in PwMS, but treatment modalities' variability among the included studies may give rise to various interpretations. ${ }^{54}$ Furthermore, we expect different results due to the dosage of our treatment compared with those of included studies in the review and treatment administration modalities that include home-based interventions and one intervention based on telerehabilitation. Instead, our expected results are in line with what was found by Nascimento et al in their systematic review regarding fatigue, quality of life and balance. ${ }^{55}$ Making the rehabilitative session more engaging for patients may increase involvement and adherence in the rehabilitation process. Increased participation and motivation were already being observed in PwMS treated with gamified training. ${ }^{53}{ }^{56}$ Traditional rehabilitation approaches is often repetitive and boring, decreasing patient's interest and exercise participation. However, patients' satisfaction represents one of the key features for treatment adherence and rehabilitation success, particularly in PwMS. ${ }^{57}$ Virtual reality-based therapy has been proposed to overcome the drawbacks 
of conventional rehabilitation, providing augmented feedback during training that contribute to a more effective motor learning. ${ }^{58}$ Active video games may offer an enriched environment useful for subjects with neuropsychological disorders, like attentional deficit or impaired alertness. Exergaming has recently been studied as an effective strategy to improve dual-task performance in people with neurological disabilities. ${ }^{59}$ Indeed, active video games not only engage patients in motor activities, but it simultaneously require subjects to use cognitive abilities for managing inputs into an enriched environment. ${ }^{5}$ Therefore, we expect to observe greater modifications on cognitive and psychological evaluation of people who received rehabilitation through video game platforms. Recently, gaming consoles introduced in clinical and research settings may represent a low-cost opportunity of delivering virtual-reality training. For this reason, the use of VGT may be delivered at home, promoting selfmanagement strategies to improve mobility function and long-term outcomes. Our study may have several limitations. First, the absence of evaluation over 3 months could not give any information about the long-term effects of active video game training. Second, we will not use any instrument to assess patients' satisfaction with the experimental treatment. Third, any neuroimaging technique will be used to show the possible neuroplastic changes in the brain due to VGT. Finally, we will not study the effects of combined treatment of VGT and other rehabilitative techniques for balance and mobility, despite combining treatments seems to augment training efficacy and boost effects of a single approach. ${ }^{22}$ Further studies should consider these possible limitations and confirm the results related to physical and neuropsychological outcomes.

Contributors SS, GZ, AB, NB and GS conceptualised the protocol. AB, GM and GF drafted the manuscript. AB, GF, GM, NB, SS and GS read and corrected the manuscript. All authors have read and approved the final manuscript.

Funding This research was supported by the Multiple Sclerosis Italian Society (Grant 2018/R/19).

Competing interests None declared.

Patient consent for publication Not applicable.

Provenance and peer review Not commissioned; externally peer reviewed.

Supplemental material This content has been supplied by the author(s). It has not been vetted by BMJ Publishing Group Limited (BMJ) and may not have been peer-reviewed. Any opinions or recommendations discussed are solely those of the author(s) and are not endorsed by BMJ. BMJ disclaims all liability and responsibility arising from any reliance placed on the content. Where the content includes any translated material, BMJ does not warrant the accuracy and reliability of the translations (including but not limited to local regulations, clinical guidelines, terminology, drug names and drug dosages), and is not responsible for any error and/or omissions arising from translation and adaptation or otherwise.

Open access This is an open access article distributed in accordance with the Creative Commons Attribution Non Commercial (CC BY-NC 4.0) license, which permits others to distribute, remix, adapt, build upon this work non-commercially, and license their derivative works on different terms, provided the original work is properly cited, appropriate credit is given, any changes made indicated, and the use is non-commercial. See: http://creativecommons.org/licenses/by-nc/4.0/.

\section{ORCID iD}

Sofia Straudi http://orcid.org/0000-0002-2061-9922
REFERENCES

1 Organization WH, Federation MSI. Atlas : multiple sclerosis resources in the world 2008. World Health Organization, 2008.

2 Khan F, Amatya B. Rehabilitation in multiple sclerosis: a systematic review of systematic reviews. Arch Phys Med Rehabil 2017;98:353-67.

3 Cameron $\mathrm{MH}$, Nilsagard Y. Balance, gait, and falls in multiple sclerosis. Handb Clin Neurol 2018;159:237-50.

4 Martin CL, Phillips BA, Kilpatrick TJ, et al. Gait and balance impairment in early multiple sclerosis in the absence of clinical disability. Mult Scler 2006;12:620-8.

5 Prosperini L, Sbardella E, Raz E, et al. Multiple sclerosis: white and gray matter damage associated with balance deficit detected at static posturography. Radiology 2013;268:181-9.

6 Cameron $\mathrm{MH}$, Lord S. Postural control in multiple sclerosis: implications for fall prevention. Curr Neurol Neurosci Rep 2010;10:407-12.

7 Chiaravalloti ND, DeLuca J. Cognitive impairment in multiple sclerosis. Lancet Neurol 2008;7:1139-51.

8 Giazkoulidou A, Messinis L, Nasios G. Cognitive functions and socia cognition in multiple sclerosis: an overview. Hell J Nucl Med 2019;22 Suppl:102-10.

9 Chalah MA, Ayache SS. Deficits in social cognition: an unveiled signature of multiple sclerosis. J Int Neuropsychol Soc 2017;23:266-86.

10 Saverino A, Waller D, Rantell K, et al. The role of cognitive factors in predicting balance and fall risk in a Neuro-Rehabilitation setting. PLoS One 2016;11:e0153469.

11 Negahban H, Mofateh R, Arastoo AA, et al. The effects of cognitive loading on balance control in patients with multiple sclerosis. Gait Posture 2011;34:479-84.

12 Song $\mathrm{J}-\mathrm{H}$. The role of attention in motor control and learning. Curr Opin Psychol 2019;29:261-5.

13 Leone C, Patti F, Feys P. Measuring the cost of cognitive-motor dual tasking during walking in multiple sclerosis. Mult Scler 2015;21:123-31.

14 Chamard Witkowski L, Mallet M, Bélanger M, et al. CognitivePostural interference in multiple sclerosis. Front Neurol 2019;10:913.

15 Ruggieri S, Fanelli F, Castelli L, et al. Lesion symptom map of cognitive-postural interference in multiple sclerosis. Mult Scler 2018;24:653-62.

16 Sosnoff JJ, Wajda DA, Sandroff BM, et al. Dual task training in persons with multiple sclerosis: a feasability randomized controlled trial. Clin Rehabil 2017;31:1322-31.

17 Ghai S, Ghai I, Effenberg AO. Effects of dual tasks and Dual-task training on postural stability: a systematic review and meta-analysis. Clin Interv Aging 2017;12:557-77.

18 Rooney S, Ozkul C, Paul L. Correlates of Dual-task performance in people with multiple sclerosis: a systematic review. Gait Posture 2020;81:172-82.

19 Mulder T, Zijlstra W, Geurts A. Assessment of motor recovery and decline. Gait Posture 2002;16:198-210.

20 Laver KE, Lange B, George S, et al. Virtual reality for stroke rehabilitation. Cochrane Database Syst Rev 2017;11:CD008349.

21 Forsberg A, Nilsagård Y, Boström K. Perceptions of using videogames in rehabilitation: a dual perspective of people with multiple sclerosis and physiotherapists. Disabil Rehabil 2015;37:338-44.

22 Maggio MG, Russo M, Cuzzola MF, et al. Virtual reality in multiple sclerosis rehabilitation: a review on cognitive and motor outcomes. $J$ Clin Neurosci 2019;65:106-11.

23 Schaeffer E, Busch J-H, Roeben B, et al. Effects of Exergaming on attentional deficits and Dual-Tasking in Parkinson's disease. Front Neurol 2019;10:646.

24 Parra-Moreno M, Rodríguez-Juan JJ, Ruiz-Cárdenas JD. Use of commercial video games to improve postural balance in patients with multiple sclerosis: a systematic review and meta-analysis of randomised controlled clinical trials. Neurologia 2018. doi:10.1016/j. nrl.2017.12.001. [Epub ahead of print: 07 Mar 2018].

25 Brichetto G, Spallarossa P, de Carvalho MLL. The effect of Nintendo $®$ Wiiß on balance in people with multiple sclerosis: a pilot randomized control study. Mult Scler Houndmills Basingstoke Engl 2013;19:1219-21.

26 Pitteri M, Dapor C, Ziccardi S, et al. Visual-Attentional load unveils slowed processing speed in multiple sclerosis patients: a pilot study with a tablet-based Videogame. Brain Sci 2020;10. doi:10.3390/ brainsci10110871. [Epub ahead of print: 18112020 ].

27 Bove R, Rowles W, Zhao C, et al. A novel in-home digital treatment to improve processing speed in people with multiple sclerosis: a pilot study. Mult Scler 2021;27:778-89. 
28 Chan A-W, Tetzlaff JM, Gøtzsche PC, et al. Spirit 2013 explanation and elaboration: guidance for protocols of clinical trials. BMJ 2013;346:e7586.

29 Kurtzke JF. Rating neurologic impairment in multiple sclerosis: an expanded disability status scale (EDSS). Neurology 1983;33:1444-52.

30 Kalron A, Dolev M, Givon U. Further construct validity of the timed Up-and-Go test as a measure of ambulation in multiple sclerosis patients. Eur J Phys Rehabil Med 2017;53:841-7.

31 Beatty WW, Goodkin DE. Screening for cognitive impairment in multiple sclerosis. An evaluation of the Mini-Mental state examination. Arch Neurol 1990;47:297-301.

32 Schulz KF, Altman DG, Moher D, et al. Consort 2010 statement: updated guidelines for reporting parallel group randomised trials. BMJ 2010;340:c332.

33 Straudi S, Severini G, Sabbagh Charabati A, et al. The effects of video game therapy on balance and attention in chronic ambulatory traumatic brain injury: an exploratory study. BMC Neurol 2017;17:86.

34 Nilsagård YE, Forsberg AS, von Koch L. Balance exercise for persons with multiple sclerosis using Wii games: a randomised, controlled multi-centre study. Mult Scler 2013;19:209-16.

35 Bonnechère $B$, Jansen $B$, Omelina $L$, et al. The use of commercial video games in rehabilitation: a systematic review. Int J Rehabil Res 2016;39:277-90.

36 Cano Porras D, Siemonsma P, Inzelberg R, et al. Advantages of virtual reality in the rehabilitation of balance and gait: systematic review. Neurology 2018;90:1017-25.

37 Eftekharsadat B, Babaei-Ghazani A, Mohammadzadeh M, et al. Effect of virtual reality-based balance training in multiple sclerosis. Neurol Res 2015;37:539-44.

38 Mañago MM, Cameron M, Schenkman M. Association of the dynamic gait index to fall history and muscle function in people with multiple sclerosis. Disabil Rehabil 2020;42:3707-12.

39 Moore M, Barker K. The validity and reliability of the four square step test in different adult populations: a systematic review. Syst Rev 2017;6:187.

40 Soke F, Eldemir S, Ozkan T, et al. The functional reach test in people with multiple sclerosis: a reliability and validity study. Physiother Theory Pract 2021:1-15.

41 Hobart J, Lamping D, Fitzpatrick R, et al. The multiple sclerosis impact scale (MSIS-29): a new patient-based outcome measure. Brain 2001:124:962-73.

42 Marengo D, Rosato R, Gamberini G, et al. Examining the validity of the multiple-sclerosis walking Scale-12 with Rasch analysis: results from an Italian study. Mult Scler Relat Disord 2019;36:101400.

43 Rooney S, McFadyen DA, Wood DL, et al. Minimally important difference of the fatigue severity scale and modified fatigue impact scale in people with multiple sclerosis. Mult Scler Relat Disord 2019;35:158-63.

44 Coghe G, Corona F, Marongiu E, et al. Fatigue, as measured using the modified fatigue impact scale, is a predictor of processing speed improvement induced by exercise in patients with multiple sclerosis: data from a randomized controlled trial. J Neurol 2018;265:1328-33.

45 Sacco R, Santangelo G, Stamenova S, et al. Psychometric properties and validity of Beck depression inventory II in multiple sclerosis. Eur J Neurol 2016;23:744-50.

46 Santangelo G, Sacco R, Siciliano M, et al. Anxiety in multiple sclerosis: psychometric properties of the State-Trait anxiety inventory. Acta Neurol Scand 2016;134:458-66.

47 Zimmermann P, Fimm B. A test battery for attentional performance. In: Applied Neuropsychology of Attention. Theory, Diagnosis and Rehabilitation 2002:110-51.

48 Coghe G, Pilloni G, Zucca E, et al. Exploring cognitive motor interference in multiple sclerosis by the visual Stroop test. Mult Scler Relat Disord 2018;22:8-11.

49 Benedict RH, DeLuca J, Phillips G, et al. Validity of the symbol digit modalities test as a cognition performance outcome measure for multiple sclerosis. Mult Scler 2017;23:721-33.

50 Sun R, Moon Y, McGinnis RS, et al. Assessment of postural sway in individuals with multiple sclerosis using a novel wearable inertia sensor. Digit Biomark 2018;2:1-10.

51 Severini G, Straudi S, Pavarelli C, et al. Use of Nintendo Wii balance board for posturographic analysis of multiple sclerosis patients with minimal balance impairment. J Neuroeng Rehabil 2017;14:19.

52 Cohen J. Statistical power analysis for the behavioral sciences. 2nd ed. New York: Routledge, 1988.

53 Dalmazane M, Gallou-Guyot M, Compagnat M, et al. Effects on gait and balance of home-based active video game interventions in persons with multiple sclerosis: a systematic review. Mult Scler Relat Disord 2021;51:102928.

54 Casuso-Holgado MJ, Martín-Valero R, Carazo AF, et al. Effectiveness of virtual reality training for balance and gait rehabilitation in people with multiple sclerosis: a systematic review and meta-analysis. Clin Rehabil 2018;32:1220-34.

55 Nascimento AS, Fagundes CV, Mendes FADS, et al. Effectiveness of virtual reality rehabilitation in persons with multiple sclerosis: a systematic review and meta-analysis of randomized controlled trials. Mult Scler Relat Disord 2021;54:103128.

56 Sokolov AA, Grivaz P, Bove R. Cognitive deficits in multiple sclerosis: recent advances in treatment and neurorehabilitation. Curr Treat Options Neurol 2018;20:53.

57 Jeong IC, Liu J, Finkelstein J. Factors affecting adherence with Telerehabilitation in patients with multiple sclerosis. Stud Health Technol Inform 2019;257:189-93.

58 Meca-Lallana V, Prefasi D, Alabarcez W, et al. A pilot study to explore patient satisfaction with a virtual rehabilitation program in multiple sclerosis: the RehabVR study protocol. Front Neurol 2020;11:900.

59 Mura G, Carta MG, Sancassiani F, et al. Active exergames to improve cognitive functioning in neurological disabilities: a systematic review and meta-analysis. Eur J Phys Rehabil Med 2018;54:450-62. 